Research Paper

\title{
Efficacy of Honeycomb TCP-induced Microenvironment on Bone Tissue Regeneration in Craniofacial Area
}

\author{
Satoko Watanabe, ${ }^{1}$ Kiyofumi Takabatake, ${ }^{2}$ Hidetsugu Tsujigiwa, ${ }^{3}$ Toshiyuki Watanabe, ${ }^{1}$ Eijiro Tokuyama, ${ }^{1}$ \\ Satoshi Ito ${ }^{2}$, Hitoshi Nagatsuka, ${ }^{2}$ Yoshihiro Kimata ${ }^{\circledR}$ \\ 1. Department of Plastic and Reconstructive Surgery, Okayama University, Graduate School of Medicine, Dentistry and Pharmaceutical Sciences, Okayama, \\ Japan. \\ 2. Department of Oral Pathology and Medicine, Okayama University, Graduate School of Medicine, Dentistry and Pharmaceutical Sciences, Okayama, Japan. \\ 3. Department of Life Science, Faculty of Science, Okayama University Science, Japan. \\ $\square$ Corresponding authors: Kiyofumi Takabatake, e-mail:gmd422094@s.okayama-u.ac.jp, TEL: +81-86-235-6652, FAX: +81-86-235-6654. Hidetsugu Tsujigiwa, \\ e-mail:tsuji@dls.ous.ac.jp, TEL/FAX: +81-86-256-9523
}

๑ Ivyspring International Publisher. Reproduction is permitted for personal, noncommercial use, provided that the article is in whole, unmodified, and properly cited. See http://ivyspring.com/terms for terms and conditions.

Received: 2016.03.17; Accepted: 2016.05.18; Published: 2016.06.01

\begin{abstract}
Artificial bone materials that exhibit high biocompatibility have been developed and are being widely used for bone tissue regeneration. However, there are no biomaterials that are minimally invasive and safe. In a previous study, we succeeded in developing honeycomb $\beta$-tricalcium phosphate $(\beta-T C P)$ which has through-and-through holes and is able to mimic the bone microenvironment for bone tissue regeneration. In the present study, we investigated how the difference in hole-diameter of honeycomb $\beta$-TCP (hole-diameter: 75, 300, 500, and $1600 \mu \mathrm{m}$ ) influences bone tissue regeneration histologically. Its osteoconductivity was also evaluated by implantation into zygomatic bone defects in rats. The results showed that the maximum bone formation was observed on the $\beta$-TCP with hole-diameter $300 \mu \mathrm{m}$, included bone marrow-like tissue and the pattern of bone tissue formation similar to host bone. Therefore, the results indicated that we could control bone tissue formation by creating a bone microenvironment provided by $\beta$-TCP. Also, in zygomatic bone defect model with honeycomb $\beta$-TCP, the result showed there was osseous union and the continuity was reproduced between the both edges of resected bone and $\beta$-TCP, which indicated the zygomatic bone reproduction fully succeeded. It is thus thought that honeycomb $\beta$-TCP may serve as an excellent biomaterial for bone tissue regeneration in the head, neck and face regions, expected in clinical applications.
\end{abstract}

Key words: honeycomb $\beta$-TCP, bone tissue regeneration, bone microenvironment, pore size, Bone morphogenetic protein-2

\section{Introduction}

Free bone transplant has been performed for bone defect reconstruction in areas such as the head and neck, face and extremities. However, problems such as sequestration and infection caused by ischemia of transferred bone tissue tend to occur in large bone defects. Although a free vascularized bone graft exhibits good synostosis because of good blood supply through anastomosis of the vascular pedicle $[1,2]$, it requires a long time to harvest the graft and the volume obtained for harvest is limited because of donor site morbidity [3-5]. Furthermore, severe complications like total graft necrosis may occur due to problems with the vascular pedicle [6]. Recently, bone tissue reconstruction performed with artificial bone has received much attention due to its low invasiveness and shorter surgical time. In addition, it has the advantage of availability of adequate volume and shape depending on the required component. However, some problems remain such as exposure and infection of material.

Three key factors are essential for the process of tissue regeneration: cells, extracellular matrix (ECM) 
and growth factors. In addition, vascularity as a nutrient source and dynamic elements influence the factors. In previous studies on ECM which is one of the important elements, various synthetic biomaterials have been developed in order to reproduce the extracellular microenvironment [7-10]. Several bioceramics having high biocompatibility like hydroxyapatite (HA), $\beta$-tricalcium phosphate $(\beta$-TCP) and calcium are already applied clinically for bone tissue regeneration [11-14]. These materials function as a scaffold on which bone cells proliferate and differentiate, at the same time the scaffold materials resorb and are replaced with new bone tissue gradually. HA is hardly or very slowly absorbed in vivo, but $\beta$-TCP is more easily absorbed compared with HA. The risk of foreign body reaction and infection of bone prosthetic material can be reduced if new bone cell infiltration and neovascularization, serving as a nutrient source, are induced into the center of the bone material; it will be replaced by bone tissue almost completely. Recently, the results from some studies have indicated that the geometric characteristics of biomaterials play an important role in neovascularization and osteoinduction, so the pore shape and size of those materials have been contrived for optimal osteogenesis [15, 16]. However, most synthetic bone materials currently on the market have coecums in those pores acting as barriers that interrupt osteogenesis and vascular development into the center of pore, thereby preventing replacement of most biomaterials by new bone tissue in the center of pores and remains a foreign body [17]. Therefore, if a large amount of artificial bone material has to be transplanted or if vascularity of the recipient site is poor, a lower graft success can be expected because of insufficient penetration and proliferation of osteocytes and vessels to the center.

We have already succeeded in developing new honeycomb $\beta$-TCP which has a through-and-through hole penetrating the material in order to overcome the problems mentioned above. It was found that histologically the honeycomb $\beta$-TCP had high biological activity, when $\beta$-TCP at varied sintering temperatures was embedded into an experimental animal model [18]. In this study, we reproduced an extracellular microenvironment using new $\beta$-TCP that contained through-and-through holes and varied pore size in each material and investigated the effect of the extracellular microenvironment formed by honeycomb $\beta$-TCP on bone tissue formation. In addition, we selected the most optimal pore size of honeycomb $\beta$-TCP and evaluated the compatibility as a material for bone tissue reconstruction when transplanted into a zygomatic bone defect in rat.

\section{Materials and methods}

\section{Preparation of honeycomb TCP containing BMP-2}

Honeycomb $\beta$-TCP was pressed in a cylindrical mold with a depth of $5 \mathrm{~mm}$, which contained through-and-through holes of diameter $75 \mu \mathrm{m}$ (75TCP), $300 \mu \mathrm{m}$ (300TCP), $500 \mu \mathrm{m}$ (500TCP), $1600 \mu \mathrm{m}$ (1600TCP). And each $\beta$-TCP was calcinated by heating to $1200{ }^{\circ} \mathrm{C}$ (Fig. 1). The detailed method of $\beta$-TCP manufacture has been described previously [18].

Each $\beta$-TCP was sterilized by autoclave, and was loaded with Bone morphogenetic protein-2 (BMP-2), which was diluted to a final contained amount of 1000 ng (1000BMP), 500 ng (500BMP), 250 ng (250BMP), $125 \mathrm{ng}$ (125BMP), and $0 \mathrm{ng}$ (0BMP) in Matrigel ${ }^{\circledR}$ (BD Bioscience). For BMP-2 loading, we centrifuged TCP and Matrigel ${ }^{\circledR}$ added with BMP-2 $\left(4{ }^{\circ} \mathrm{C}, 10000 \mathrm{rpm}, 5\right.$ $\mathrm{min})$. In the control group, we centrifuged TCP and Matrigel ${ }^{\circledR}$ without BMP-2.

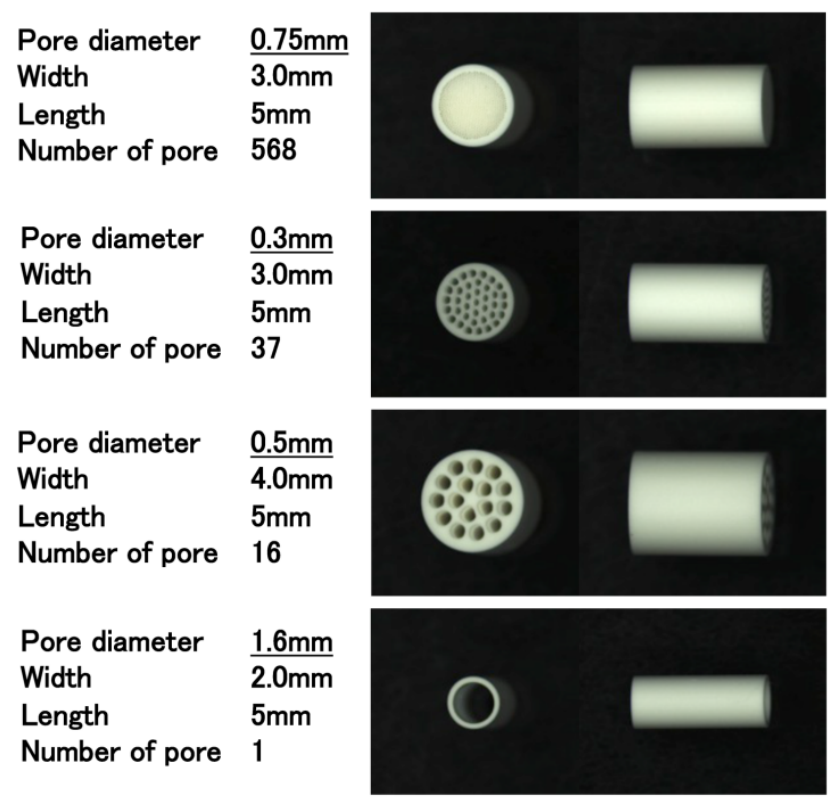

Fig. I. Images of honeycomb $\beta$-TCP used in this experiment.

\section{Animals and implantation procedure}

Four-week-old healthy male Wister rats were used in this experiment. All experiments in this study were performed in accordance with the Policy on the Care and Use of the Laboratory Animals, Okayama University and approved by the Animal Care and Use Committee, Okayama University, and all surgical procedures were performed under general anesthesia, in a pain-free state.

To investigate the osteoconductivity of honeycomb $\beta$-TCP, the animals were randomly divided into 20 groups: different holes of honeycomb 
$\beta$-TCP (4 types) $\times$ different amount of BMP-2 (5 conditions), total 20 groups.

Wistar male rats were anesthetized intraperitoneally with ketamine hydrochloride (75 $\mathrm{mg} / \mathrm{kg}$ body weight), medetomidine hydrochloride $(0.5 \mathrm{mg} / \mathrm{kg}$ body weight $)$ and atipamezole hydrochloride (1 $\mathrm{mg} / \mathrm{kg}$ body weight) was injected subcutaneously when awakening. The region of hip from femoral region was shaved, cleaned with $70 \%$ alcohol and iodine, and cut $10 \mathrm{~mm}$ by blunt dissection to form $8 \mathrm{~mm}$ intramuscular pockets. Each sample was implanted carefully with tweezers in the intramuscular pockets and sutured. The animals were killed with an overdose of ether at 3 weeks after implantation. For histological observations, implanted $\beta$-TCPs were fixed in perfusion fixation by $4 \%$ paraformaldehyde (PFA).

\section{Zygomatic bone reproduction of honeycomb $\beta$-TCP}

To evaluate the osteoinductive ability of the $\beta$-TCP in a bone defect, we implanted the samples, which were the most osteoconductive and formed bone marrow-like tissue in the intramuscular experiment, into the rat zygomatic bone defect. A method for preparation of rat zygomatic bone defect model is described below. At first, skin incision about $8 \mathrm{~mm}$ was made just above the zygomatic bone, and the masseter muscle that adhered to the zygomatic bone was completely separated from the bone, the zygomatic bone was exposed entirely. Next, the zygomatic bone periosteum was incised by a surgical knife and was completely peeled from the zygomatic bone, and bone was totally cut in two places using scissors in front of the arch of zygomatic bone to create $5 \mathrm{~mm}$ bone defect.

Then, $\beta$-TCP alone and $\beta$-TCP added with BMP were implanted into this bone defect with the through-and-through holes of $\beta$-TCP and the long axis of the bone defects was parallel. And completely zygomatic bone defect without $\beta$-TCP were prepared as a control. For each groups, four to five Wister rats were used. Each $\beta$-TCP was embedded 3 weeks later, embedded tissues were fixed with $4 \%$ PFA reflux fixation, and we investigated the specimens by micro CT and histology.

\section{Histological procedure and Immunohistoche- mical staining of osteopontin}

The specimens were decalcified in 10\% ethylenediaminetetraacetic acid for 3 weeks. They were embedded in paraffin, and sectioned to 5- $\mu \mathrm{m}$ thickness. Sections were chemically stained with hematoxylin and eosin $(\mathrm{H} \& \mathrm{E})$, toluidine blue and observed histologically.
Osteopontin (OPN) is a noncollagenous protein that is produced in abundance in the bone extracellular matrix by the osteoblasts responsible for bone formation. Therefore, the presence of this bone protein was investigated.

Sections were deparaffinized, rehydrated, and incubated in proteinase $\mathrm{K}$ for $15 \mathrm{~min}$ at room temperature. Endogenous peroxidase was blocked using a $0.3 \%$ hydrogen peroxide solution in methanol for $20 \mathrm{~min}$. Nonspecific binding sites were blocked with 10\% normal rabbit antiserum (Vector Laboratories, Burlingame, CA) for $10 \mathrm{~min}$. The sections were incubated with monoclonal antibodies against rat OPN (Immuno-Biological Laboratories, Gunma, Japan) following the Vectastain ABC Mouse Kit method (Vector Laboratories, Burlingame, CA). The principal steps were as follows: (1) incubation with primary antibodies at a dilution of 1:50; (2) incubation with secondary anti-mouse IgG antibodies at a dilution of 1:200 for $30 \mathrm{~min}$; (3) incubation with avidin-biotin-peroxidase complex (ABC; Vector Laboratories, Burlingame, CA) at a dilution of 1:50 for 30 min; (4) treatment with Diaminobenzidine color development and nuclear counterstaining with Mayer's hematoxylin. Staining was visualized using a light microscope. The control sections were processed in the same way but in the absence of the primary antibodies.

\section{Bone and cartilage tissue formation evaluation by area measurement}

HE-stained specimens were taken using a Nikon Elipse 80i microscope (Teknooptik AB, Huddinge, Sweden), equipped with an Easy Image 2000 system (Teknooptik AB) using 103 to 403 lenses. In HE staining specimens (100× magnification), we investigated the image taken at a total of 5 fields (5 fields: at the center, both ends, and the center of the center and both ends) using Image J1.47v [developed by Wayne Rasband, the National Institute of Health (NHS)]. In each field, we measured the total area of bone formation in $\beta$-TCP holes and the area of $\beta$-TCP holes and we calculated the ratio of area of bone area in $\beta$-TCP holes to determine the average of the 5 fields. The obtained average value was compared in each group, the rate of bone formation and cartilage formation were compared for different pore size and BMP concentration.

\section{Micro CT}

In the zygomatic bone defect model, the head specimens after fixation were taken with micro CT (Hitachi Aloka Latheta LCT200), and the resulting DICOM data was reconstructed three-dimensionally by using the workstation and software (AZE 
VirtualPlace Lexus64). Then, we assessed bone tissue formation in the image.

\section{Results}

\section{The effect of honeycomb $\beta$-TCP on bone and cartilage tissue formation}

The incidence rate of bone and cartilage tissue formation depending on the amount of BMP is shown

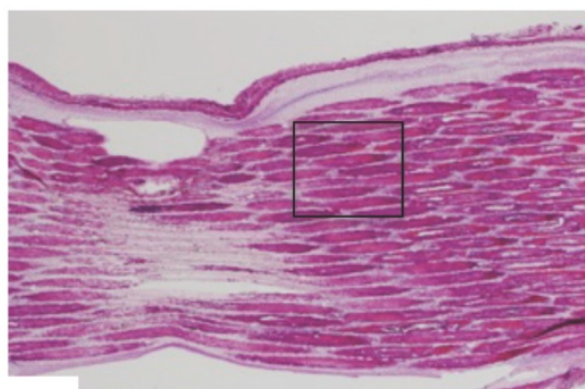

\section{a}
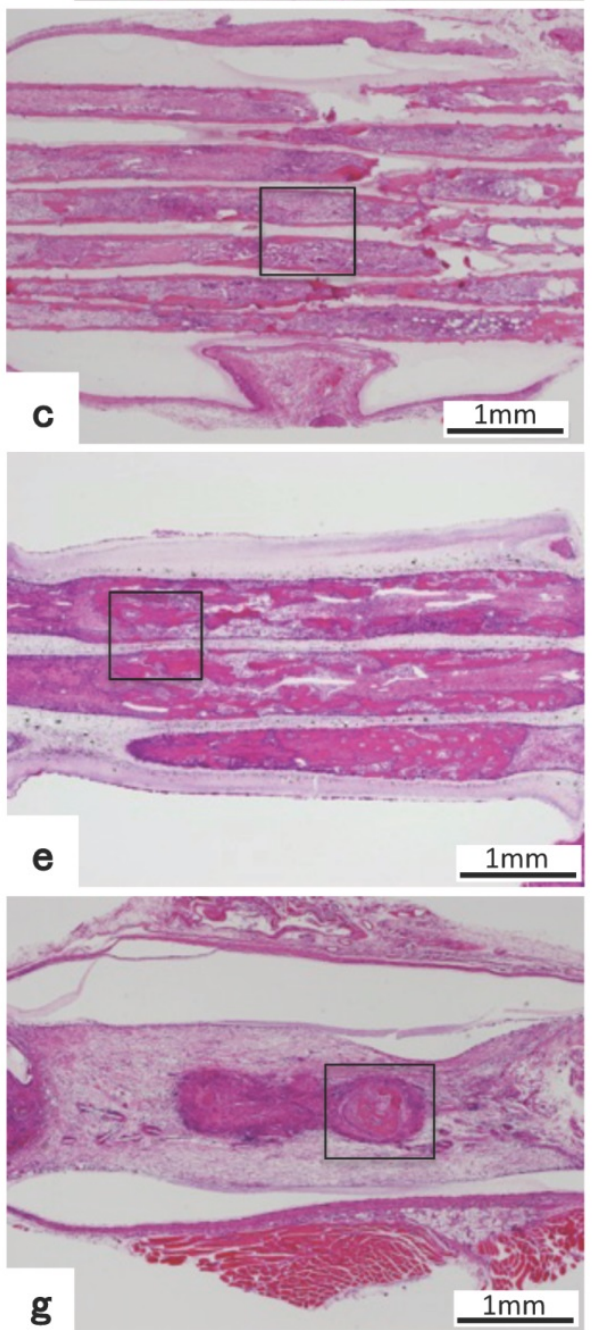

in Table 1. In the control group, bone formation was observed in all samples with 125BMP, but the incidence rates of that were not so high. The incidence rates were getting higher, as the amount of BMP was increased and all the samples with 1000BMP, except 1600TCP, showed bone formation. 1600TCP seemed likely to promote less bone formation than others regardless of the amount of BMP.
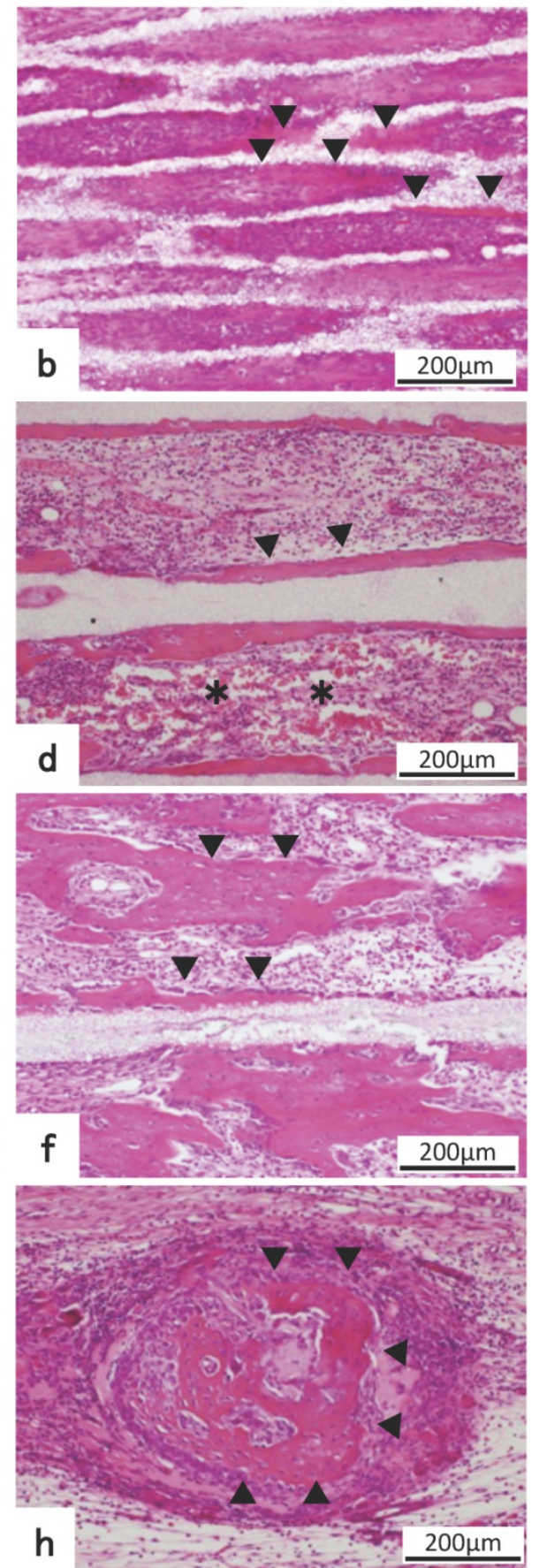

Fig. 2. HE staining images of each honeycomb $\beta$-TCP with added $1000 \mathrm{ng}$ BMP-2 3 weeks after implantation. a) Lower-magnification images of $75 T C P$. b) Higher-magnification image of corresponding outline area in (a). c) Lower-magnification images of 300TCP. d) Higher-magnification image of corresponding outline area in (c). e) Lower-magnification images of 500TCP. f) Higher-magnification image of corresponding outline area in (e). g) Lower-magnification images of 1600TCP. h) Higher-magnification image of corresponding outline area in $(\mathrm{g})$. Bone formation pattern in each pore size TCP was different, bone tissue filled in the holes of 75TCP. Bone formation was observed in 300TCP adding to the inner wall, and also bone marrow-like tissue was observed in some parts. In 500TCP, cancellous bone-like bone tissue was observed, and in $1600 T C P$ bone tissue formation was observed in the center of TCP hole. Bone tissue is indicated by arrowheads, and bone marrow-like tissue is indicated by an asterisk. 
There were quite a few samples that showed cartilage formation except 75TCP. Even though cartilage formation was observed in some samples, cartilage tissue extended to just a part of them and the inner lumen of TCP was not totally replaced by cartilage tissue. In $1600 \mathrm{TCP}$, with any BMP, cartilage tissue was not observed. However, the 75TCP group was different from the others, as there was a high incidence rate of cartilage tissue formation not only in samples with large amounts of BMP but also in samples with small amounts (Table 1).

\section{Histological findings as vital reaction on honeycomb $\beta$-TCP}

In 75TCP with 1000BMP, new bone formation was seen with fibroblast like cells filling the inner part of hole, but there was a lack of vascularization and little infiltration of the inflammatory cells. In a part of them, cartilage tissue spread through the hole as if they filled the lumen and some findings indicated calcification of cartilage tissue. Although the pattern of bone tissue formation was similar to that of cartilage regardless of the amount of BMP, cartilage formation with a few bone tissues was remarkably observed in some samples with a small amount of BMP (Fig. 2 a, b).

In 300TCP with 1000BMP, bone tissue formation, differing from that in 75TCP, was observed on the $\beta$-TCP and also on the $\beta$-TCP inner wall, but there were few cancellous bone-like trabeculae inside the hole. Bone formation was present up to the center of

Table 1. The incidence rate of bone and cartilage tissue formation depending on the amount of BMP.

\begin{tabular}{lrrrrc}
\multicolumn{3}{l}{ Incidence Rate of Bone Formation in Each Type of Honeycomb $\beta$-TCP } \\
\cline { 2 - 6 } & & \multicolumn{5}{c}{$\beta$-TCP } & pore size $(\mu \mathrm{m})$ \\
\hline \multirow{2}{*}{ BMP-2(ng) } & 0 & $0 / 5$ & $0 / 5$ & $0 / 5$ & $0 / 5$ \\
& 125 & $4 / 5$ & $3 / 5$ & $3 / 5$ & $1 / 6$ \\
& 250 & $3 / 6$ & $5 / 5$ & $4 / 5$ & $2 / 5$ \\
& 500 & $4 / 5$ & $4 / 5$ & $5 / 6$ & $1 / 6$ \\
1000 & $5 / 5$ & $6 / 6$ & $6 / 6$ & $3 / 7$ \\
\hline
\end{tabular}

Number of bone induced samples/ Total number of samples in each group

\begin{tabular}{rrrrrr}
\multicolumn{2}{l}{ Incidence Rate of Cartilage Formation in Each Type of Honeycomb $\beta$-TCP } \\
\cline { 2 - 6 } & & \multicolumn{5}{c}{$\beta-\mathrm{TCP}$} & pore size $(\mu \mathrm{m})$ \\
\hline \multirow{2}{*}{ BMP-2 (ng) } & 0 & $0 / 5$ & $0 / 5$ & $0 / 5$ & $0 / 5$ \\
& 125 & $4 / 5$ & $0 / 5$ & $0 / 5$ & $0 / 6$ \\
250 & $3 / 6$ & $1 / 5$ & $1 / 5$ & $0 / 5$ \\
500 & $5 / 5$ & $1 / 5$ & $0 / 6$ & $0 / 6$ \\
1000 & $5 / 5$ & $0 / 6$ & $1 / 6$ & $0 / 7$ \\
\hline
\end{tabular}

Number of cartilage induced samples/ Total number of samples in each group
$\beta$-TCP and there were numerous osteoblasts arrayed in a single line around the bone matrix, which suggest that bone-forming activity was high. Additionally, a large amount of capillaries were seen piercing through the hole surrounded by bone tissue, and also in the center part of $\beta$-TCP. Bone marrow-like tissue which had many blood cells was observed in part of the vessel lumen. In 300TCP+1000BMP samples, the pattern of bone tissue formation was similar regardless of the amount of BMP. There were some osteoclasts and some findings showed that $\beta$-TCP was absorbed and replaced by bone tissue (Fig. 2 c,d).

$500 \mathrm{TCP}$ with $1000 \mathrm{ng}$ had a similar pattern of bone formation as 300TCP; in addition, there were numerous newly beam-shape cancellous bone tissues. However, there were not any kind of tissues that looked like bone marrow tissue in the area surrounded by bone tissue (Fig. $2 \mathrm{e}, \mathrm{f}$ ).

In $1600 \mathrm{TCP}$, the pattern of bone formation was different from other pore size $\beta$-TCP. For $1600 \mathrm{TCP}+1000 \mathrm{BMP}$, isolated spherical new bone tissue was observed in the center of holes, but the bone tissue occupancy region was very small. Also there were fewer osteoblasts in 1600TCP than in 300TCP and 500TCP. Although blood vessels and fibroblasts were observed in the stroma surrounding new bone tissue, the, number of cells was poorer than in 300TCP or 500TCP. Vascularization and fibroblasts were observed in the interstitial tissue around the new bone tissue in 1600TCP, but those tissues had poor number of cells and consisted of coarse tissue compared to the other pore size TCP. (Fig. 2 g,h)

\section{Immunohistochemical and special staining for biological reaction of honeycomb $\beta$-TCP}

For $75 \mathrm{TCP}$, cartilage tissue filled the holes in the 125BMP group, which was the lowest concentration, and invasion of blood vessels in the holes was hardly observed. In the toluidine blue staining, cartilage matrix was stained red purple, indicating cartilage matrix-specific staining. (Fig. 3 a,b)

In 75TCP added with $125 \mathrm{ng}$ BMP-2, toluidine blue staining positive images showed cartilage-like tissue filling the holes. (Fig. 3 c,d)

Immunohistochemical staining of OPN revealed that an immature bone matrix was present in the pores of 300TCP+1000BMP and new bone tissue was observed to be added to the $\beta$-TCP and also lining the $\beta$-TCP inner wall. (Fig. 3 e,f) 

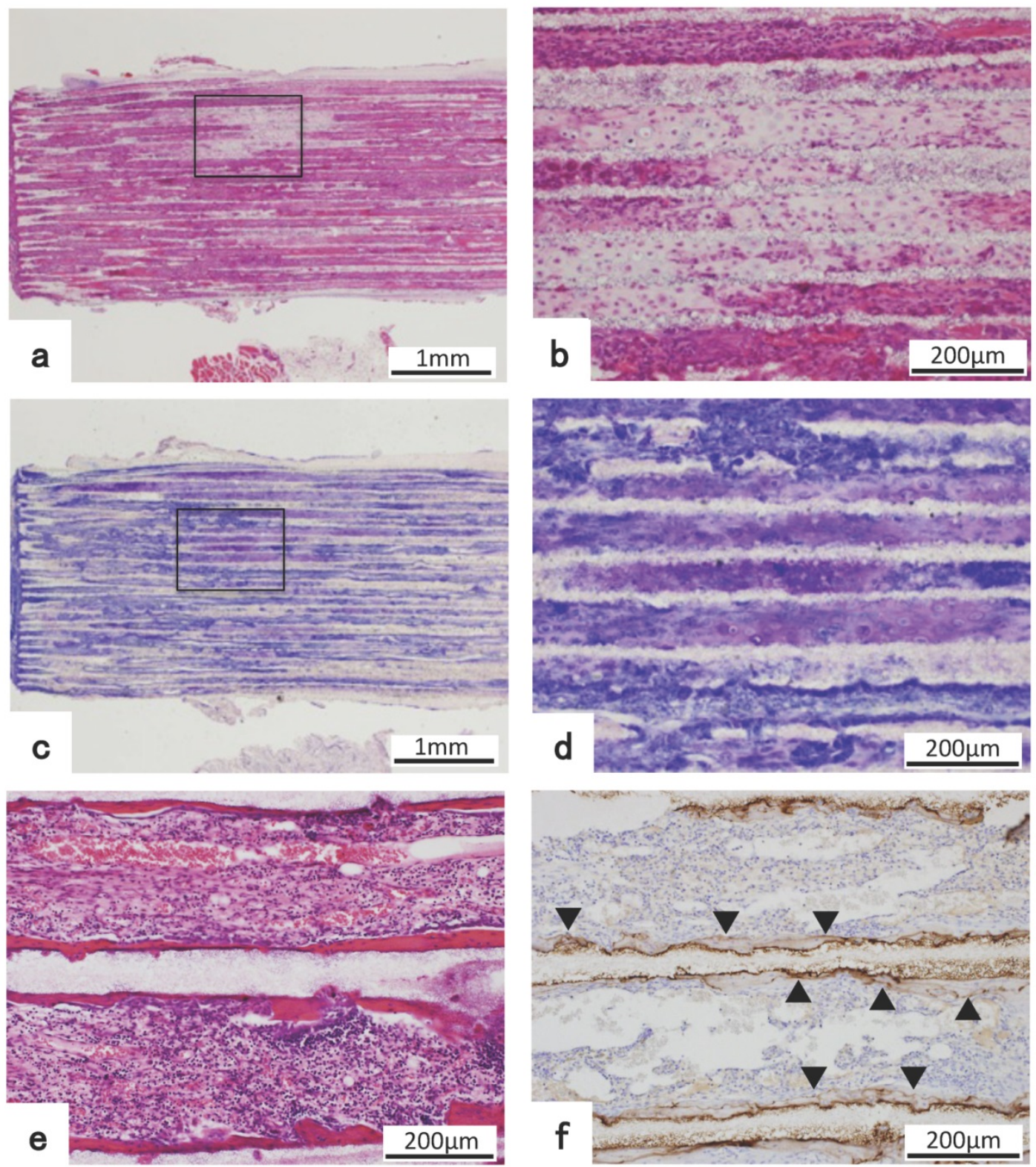

Fig 3. Toluidine blue staining and immunohistochemical staining. a) HE staining images of 75 TCP with 125 ng BMP- 2 at 3 weeks after implantation. b) Higher-magnification image of corresponding outline area in (a). c) Toluidine blue staining images of 75TCP with $125 \mathrm{ng}$ BMP-2. d) Higher-magnification image of corresponding outline area in (c). e) Immunohistochemical staining of osteopontin of 300TCP added with $1000 \mathrm{ng}$ BMP-2. f) Higher-magnification image of corresponding outline area in (e). Toluidine blue staining positive images were observed to fit the cartilage-like tissue (a-d). The positive images of osteopontin were observed in new bone tissue (e,f), and the positive images of osteopontin are indicated by arrowheads.

\section{Effect of honeycomb $\beta$-TCP pore diameter and BMP amount on bone and cartilage tissue formation}

We measured cartilage or bone formation area in honeycomb $\beta$-TCP holes, and we analyzed the relationship between BMP amount and cartilage or bone tissue formation in $\beta$-TCP holes.

Analysis revealed that bone formation was not observed in honeycomb $\beta$-TCP without BMP.

In $\beta$-TCP combined with BMP, as the amount of $\mathrm{BMP}$ was increased, bone tissue formation tended to increase regardless of TCP pore diameter.
Considering the effect of $\beta$-TCP pore size on the amount of bone tissue formation, as the pore size increased from $75 \mu \mathrm{m}$ up to $500 \mu \mathrm{m}$, bone formation amount tended to increase regardless of the amount of BMP. Bone formation in 1600TCP was very little and was hardly affected although amount of BMP was increased (Fig. 4 a).

Analysis showed that cartilage formation was not observed in honeycomb $\beta$-TCP without BMP.

In $\beta$-TCP combined with BMP, cartilage formation was observed only in 75TCP+125BMP, which was the smallest pore size and was the lowest amount of BMP. And as the amount of BMP increased 
in $75 \mathrm{TCP}$, it was observed that the area of cartilage tissue formation was decreased. In 300TCP and $500 \mathrm{TCP}$, only a small amount of cartilage tissue formation was observed regardless of the BMP amount, so relationship between BMP amount and TCP pore size was uncertain. In 1600TCP, cartilage tissue formation was hardly observed regardless of the amount of BMP. (Fig. 4 b)
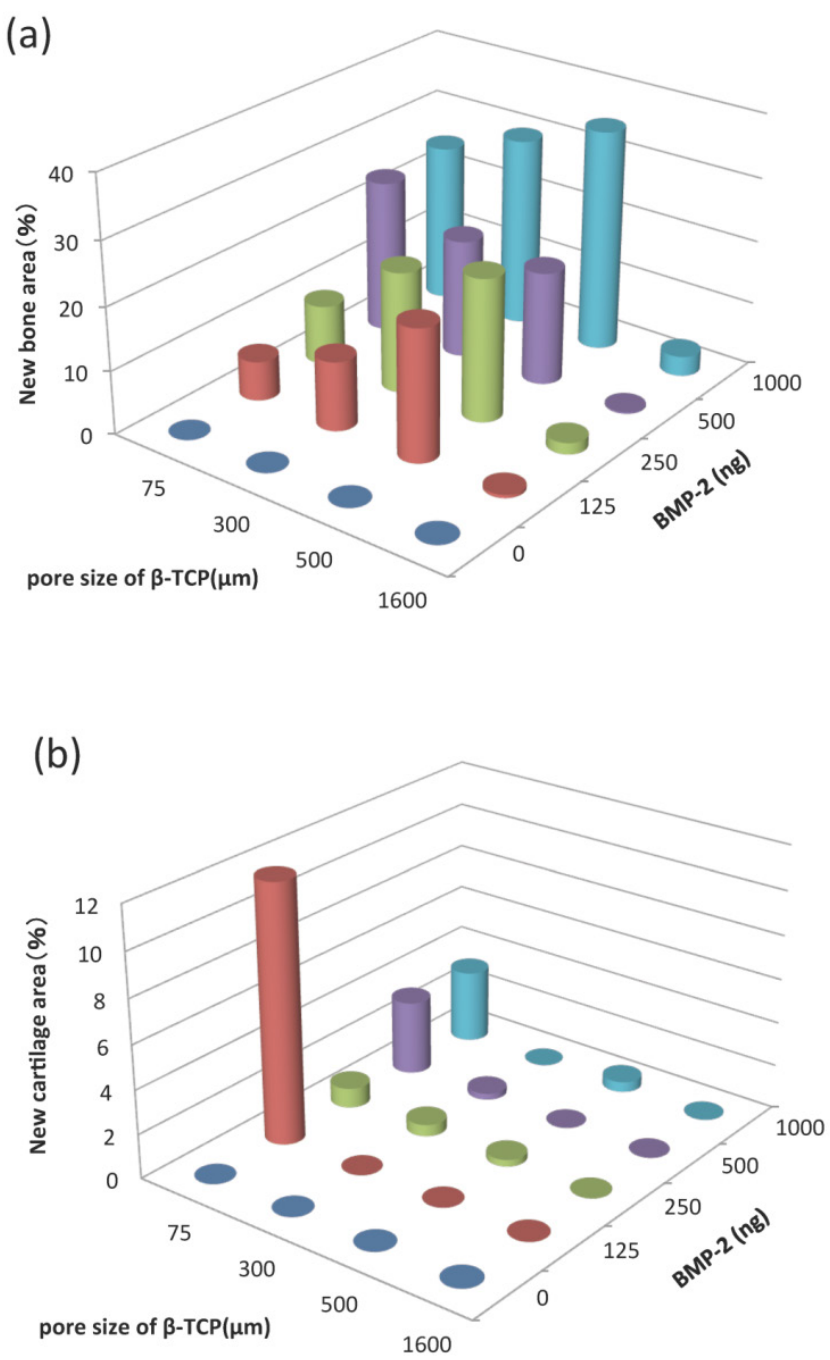

Fig 4. Graphic representation of the experimental result showing the relationship among the area ratio of bone formation in holes of $\beta$-TCP and pore size and amount of BMP-2. (a) It shows that as the amount of BMP is increased, bone tissue formation tends to increase regardless of pore size except 1600TCP. Also, it shows that as pore size becomes bigger, bone tissue formation tends to increase regardless of the amount of BMP-2 except 1600TCP. In 1600TCP, bone tissue formation decreases remarkably, which is less affected by increasing amount of BMP-2. (b) It shows that cartilage formation was observed only in 75TCP+125BMP group, which was the smallest pore size and was the lowest amount of BMP and as the amount of BMP was increased in 75TCP, cartilage tissue formation was decreased. In 300TCP and 500TCP, only a small amount of cartilage tissue formation was observed regardless of the BMP amount

\section{Micro CT findings of bone tissue regeneration in zygomatic bone defect model}

In the control group, bone tissue regeneration was not observed from the edge of bone defect to
$\beta$-TCP even 3 weeks after implantation, and the bone defect area did not change almost immediately after surgery. (Fig. $5 \mathrm{a}, \mathrm{b}, \mathrm{c}$ )

In only the $\beta$-TCP group, bone defect was maintained and new bone regeneration was not observed. There was a marginal gap between the implanted $\beta$-TCP and the bone resection stump, therefore the osseous union between $\beta$-TCP and the existing bone tissue was not clear. (Fig. 5 d,e,f)

In the $\beta-\mathrm{TCP}+\mathrm{BMP}$ group, new bone formation was observed from the edge of resected bone to $\beta$-TCP, and there was osseous union and the continuity was reproduced in those areas. In addition, new bone formation was recognized not only in the gap between the implanted $\beta$-TCP and bone resection stump, but also covering the $\beta$-TCP. (Fig. 5 g,h,i)

\section{Histological analysis on bone tissue regeneration in zygomatic bone defect model}

The pattern of bone tissue formation was similar to $\beta$-TCP implanted intramuscularly, in which bone was added to the $\beta$-TCP inner wall. Rich osteoblasts existed on the surface of new bone, and bone tissue formation had reached the center of $\beta$-TCP. (Fig. $6 a, b$ )

In both ends of $\beta$-TCP adjacent to existing bone tissue, new bone regeneration occurred from the bone resection stump, and new bone formation was combined with $\beta$-TCP. In the holes of $\beta$-TCP surrounded with bone tissue, bone marrow tissue that had rich blood vessels was observed adjacent to existing bone tissue. And on the surface of new bone tissue, osteoblasts were arranged orderly in a single-layer and osteoblasts showed endosteum-like structure. In addition, emergence of osteoclast cells was partially observed and also TCP was replaced by bone tissue by resorption. (Fig. $6 \mathrm{a}, \mathrm{c}, \mathrm{d}$ )

Immunohistochemical staining of OPN revealed that an immature bone matrix was present in the pores of $\beta-\mathrm{TCP}$, and new bone had formed adjacent to TCP in the zygomatic bone defect model experiment. (Fig. 6 e,f)

\section{Discussion}

In tissue regeneration, stem cell, scaffold and growth factor are important elements [19], so normal tissue regeneration is disturbed when any one of these factors is missing. Among these elements, artificial biomaterial plays a role as scaffold to provide an environment for proliferation and differentiation of cells. The characteristics required for ideal artificial biomaterials are not only cell proliferation and differentiation but also biocompatibility, a structure which cells are likely to invade, tissue solubility and so on. 

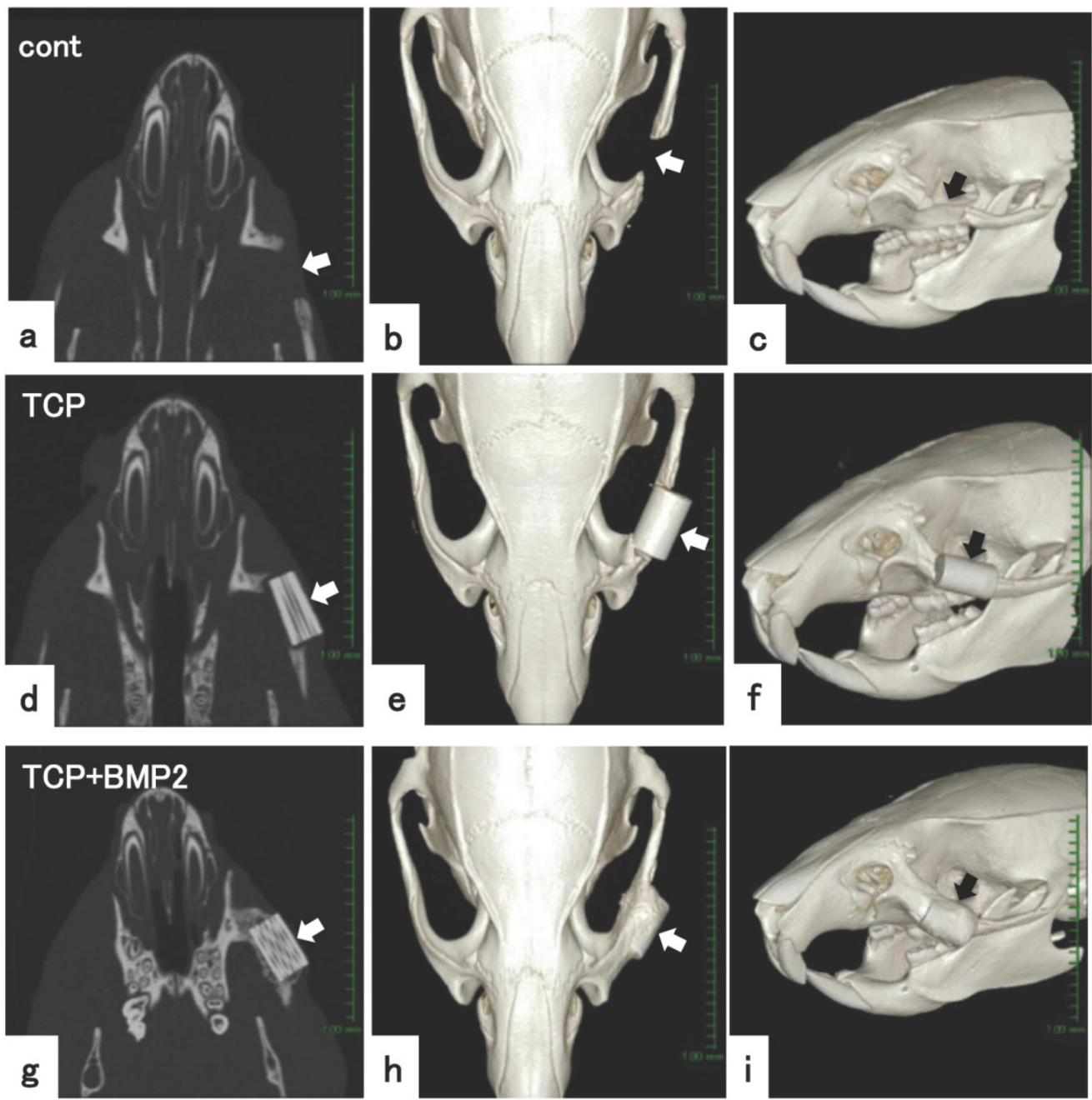

Fig 5. Analysis of bone formation on honeycomb $\beta$-TCP in micro CT image. (a-c) Micro CT images of facial bone of rat with left zygomatic bone defect. (d-f) Micro CT images of facial bone of rat with implanted 300TCP in left zygomatic bone defect site. (g-i) Micro CT images of facial bone of rat with implanted 300TCP+BMP-2 in left zygomatic bone defect site. (a,d,g) axial view of micro CT, (b,c,e,f,h,i) reconstructed 3D images of micro CT. (a-c)New bone tissue formation was not observed at bone defect site (arrow). (d-f) In TCP alone group, TCP exists at bone defect site with neither resorption nor new bone formation (arrow). (g-i) New bone formation was observed in implanted TCP+BMP- 2 group at the boundary between TCP and existing bone (arrow). The osseous union between TCP and existing bone tissue was observed (arrow).

In our study, it was shown that both bone formation rate and amount of bone formation were the greatest in $500 \mathrm{TCP}+\mathrm{BMP}-2$ in rat thigh muscle. This was followed by $300 \mathrm{TCP}+\mathrm{BMP}-2$, and in these samples, normal bone tissue-like structure that had bone marrow tissue formation was observed. Then, bone formation rate and amount tended to increase in proportion to the amount of BMP-2. These results are consistent with our previous experimental result of the ear canal bone reconstruction [18], and consistent with a previous report by Tsurug using porous granular apatite [20]. The bone formation patterns varied with $\beta$-TCP pore size, and bone tissue formation occurred so as to fill the lumen in 75TCP. In $300 \mathrm{TCP}$, the bone tissue formed on the $\beta$-TCP inner wall. In $500 \mathrm{TCP}$, similar to $300 \mathrm{TCP}$, bone tissue was formed along the inner wall of the hole, and furthermore, large amounts of cancellous bone-like tissue were also formed in $\beta$-TCP pores. In 1600TCP, solitary bone tissue was presented in the center of TCP hole. In all pore size $\beta$-TCP, the pattern of bone formation did not vary with the concentration of BMP-2. Our previous study and Kuboki et al $[15,21]$ suggested that the biological material providing the microenvironment is not actively involved in bone formation but provides only space for cell proliferation when the microenvironment of bone formation is relatively large. However, honeycomb-type hydroxyapatite that had pores of diameter 300-400 $\mu \mathrm{m}$ directly added to the bone matrix, and when it was implanted in vivo, the biomaterial functioned in bone regeneration effectively [22]. 

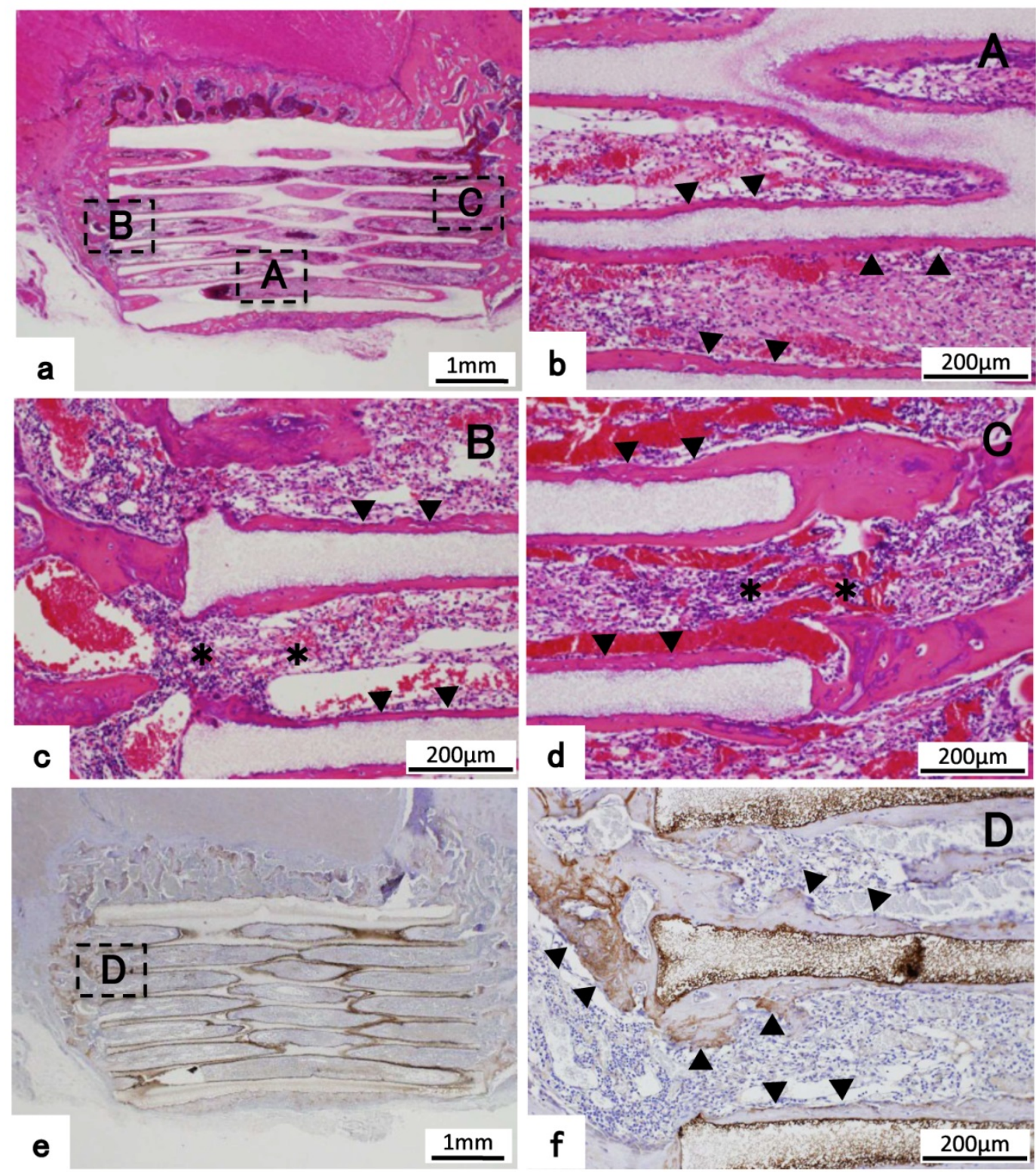

Fig 6. (a) Histological images of $300 \mathrm{TCP}+\mathrm{BMP}-2$ implanted into zygomatic defect. (b,c,d) Higher-magnification image of corresponding outline area in (a). (a-d) New bone tissue formation was observed from the bone stump, and the bone stump combined with 300TCP. New bone tissue formation was also observed in the center of TCP holes, and bone marrow-like tissue formation was observed. The positive images of osteopontin were observed in new bone tissue (e,f). Bone tissue is indicated by arrowheads, bone marrow-like tissue is indicated by asterisk, the positive images of osteopontin are indicated by arrow.

In HE images, rich blood vessels that penetrated into $\beta$-TCP holes were observed only in 300TCP and 500TCP. Many reports indicated that blood vessel formation plays an important role in regeneration of not only bone tissue but also various tissues [23-26]. Also, our study suggested that angiogenesis had a great influence on bone tissue formation. Furthermore, marked infiltration of inflammatory cells was not observed in all $\beta-\mathrm{TCP}$, and this proved that the $\beta$-TCP used in our study had extremely high biocompatibility.

The results suggest that $\beta$-TCP in this experiment has high biocompatibility, and pore size of about 300 to $500 \mu \mathrm{m} \quad \beta$-TCP provides an environment for proliferation and differentiation of cells in vivo and is the most suitable material for inducing bone tissue.

On the other hand, in this study an interesting finding was that strong chondrogenesis was shown only when using a small amount of BMP-2 in 75TCP although a little cartilage formation was observed in large amount of BMP-2 in other pore size TCP. BMP-2 is a well-known growth factor which induces bone tissue specifically. But when using both low concentration of BMP-2 and TGF known as cartilage-induce factor, the cartilage-inducing ability is higher than the case of using only TGF $[27,28]$. It is known that the BMP family is involved in normal 
cartilage tissue development [29]. Also in ectopic bone formation experiments using BMP, it has been reported that endochondral ossification-mediated cartilage formation occurs, and thus involvement of BMP-2 in cartilage formation is consistent with this experimental result. But although little cartilage formation was observed in 300TCP and 500TCP, which were recognized for strong bone formation, the most amount of cartilage tissue formation was shown in 75TCP. Therefore, it is thought that a specific microenvironment provided by 75TCP is involved in cartilage tissue formation. Further investigations are required as to what kind of environmental factors provided by 75TCP induce formation of cartilage tissue.

\section{Bone tissue regeneration in zygomatic bone defect model}

Generally, when a bone defect occurs due to a bone injury, cells are supplied from the periosteum and surrounding connective tissue, and bone tissue regeneration occurs. However, complete regeneration becomes more difficult the wider the bone defect. Therefore, various artificial biomaterials made from hydroxyapatite, calcium phosphate ceramics (TCP), polylactic acid, and titanium and so on have been developed and used clinically. In addition, many studies have reported bone tissue regeneration when using these biomaterials combined with mesenchymal stem cell [30].

But when these materials are used in bone tissue regeneration, there are still many problems such as early stage strength, efficiency of osteoinductive activity, and replacement property of bone tissue in vivo. These materials have already been used as bone substitutes in clinical practice, but in the current situation, it is difficult to obtain the regeneration on such a large total bone defect.

For the bone tissue reconstruction experiment in zygomatic bone defect model, we used 300TCP which formed bone tissue structurally similar to biological bone tissue in an ectopic experiment in which honeycomb $\beta$-TCP of each hole diameter was embedded into thigh muscle. In micro $C T$, the continuity of the zyomatic bone was not observed in the zygomatic bone resection group and in only the TCP group 3 weeks after implantation. On the other hand, new bone formation was observed in the $300 \mathrm{TCP}+\mathrm{BMP}-2$, and the continuity of the zygomatic bone tissue was recovered. In the histological observation, the pattern of bone tissue formation was almost the same as the 300TCP that was implanted into thigh muscle, and new bone formation in the inner wall of $\beta$-TCP was observed. The new bone tissue in 300TCP with BMP-2, which was accompanied with bone marrow-like tissue having rich hematopoietic cells, had continuity with the existing bone tissue and $\beta$-TCP was completely connected with the existing bone tissue. Therefore, the recovery of bone tissue continuity between $\beta$-TCP and existing bone was confirmed at the tissue level.

Many bone tissue regeneration studies using various cells have been attempted with the development of new biomaterials. However, when using a cell it is difficult to exclude the risk of tumorigenesis completely, thus bone tissue regeneration without using cells and by a simple technique is considered much more ideal. Honeycomb $\beta$-TCP has the characteristic features of excellent biocompatibility, osteoconductive ability, and bioabsorbable ability along with bone remodeling $[31,32]$. It is reported that the bioabsorbable ability of TCP is higher than hydroxyapatite which is widely used clinically [31,32]. It is thought that TCP replaces the existing bone tissue by absorption $[33,34]$.

Our study indicates that honeycomb $\beta$-TCP is an excellent artificial biomaterial because honeycomb $\beta$-TCP regenerates bone tissue that is similar to normal bone with bone marrow-like tissue and endosteum-like tissue in completely transected bone tissue. Therefore, TCP is expected to serve as a new biological material in the head and neck region.

\section{Acknowledgement}

This study was supported by a Grant-in-Aid for Scientific Research(C), 15K20309 provided by the Japan Society for Promotion of Science (JSPS).

\section{Competing Interests}

The authors have declared that no competing interest exists.

\section{References}

1. Lee KS, Park JW. Free vascularized osteocutaneous fibular graft to the tibia. Microsurgery. 1999; 19:141-147.

2. Muramatsu K, Hashimoto T, Tominaga Y, et al. Vascularized Bone Graft for Oncological Reconstruction of the Extremities: Review of the Biological Advantages. Anticancer Res. 2014; 34:2701-2707.

3. Dimitriou R, Mataliotakis GI, Angoules AG, et al. Complications following autologous bone graft harvesting from the iliac crest and using the RIA: a systematic review. Injury. 2011; 42 Suppl 2:S3-15.

4. Banwart JC, Asher MA, Hassanein RS. Iliac crest bone graft harvest donor site morbidity. A statistical evaluation. Spine (Phila Pa 1976). 1995; 20(9):1055-1060

5. Schaaf H, Lendeckel S, Howaldt HP, et al. Donor site morbidity after bone harvesting from the anterior iliac crest. Oral Surg Oral Med Oral Pathol Oral Radiol Endod. 2010; 109(1):52-58

6. Arce K, Bell RB, Potter JK, et al. Vascularized free tissue transfer for reconstruction of ablative defects in oral and oropharyngeal cancer patients undergoing salvage surgery following concomitant chemoradiation. Int J Oral Maxillofac Surg. 2012; 41(6):733-738

7. Karageorgiou V, Kaplan D. Porosity of 3D biomaterial scaffolds and osteogenesis. Biomaterials. 2005; 26(27): 5474-5491.

8. Burg KJ, Porter S, Kellam JF. Biomaterial developments for bone tissue engineering. Biomaterials. 2000; 21(23): 2347-2359.

9. Stevens M. Biomaterials for bone tissue engineering. Materials Today. 2008; 11(5):18-25.

10. Yoshikawa H, Myoui A. Bone tissue engineering with porous hydroxyapatite ceramics. J Artif Organs. 2005; 8(3):131-136. 
11. Kaltreider SA, Newman SA. Prevention and management of complications associated with the hydroxyapatite implant. Ophthal Plast Reconstr Surg. 1996; 12(1):18-31.

12. Walsh WR, Vizesi F, Michael D, et al. Beta-TCP bone graft substitutes in a bilateral rabbit tibial defect model. Biomaterials. 2008; 29(3):266-271.

13. Asaoka T, Ohtake S, Furukawa KS, et al. Development of bioactive porous alpha-TCP/HAp beads for bone tissue engineering. J Biomed Mater Res A. 2013; 101(11):3295-3300.

14. Ono I, Tateshita T, Satou M, et al. Treatment of large complex cranial bone defects by using hydroxyapatite ceramic implants. Plast Reconstr Surg. 1999; 104(2):339-349.

15. Kuboki $Y$, Jin Q, Takita H. Geometry of carriers controlling phenotypic expression in BMP-induced osteogenesis and chondrogenesis. J Bone Joint Surg Am. 2001; 83A(Suppl 1):S105-115.

16. Kuboki Y, Jin Q, Kikuchi M, et al. Geometry of artificial ECM: sizes of pores controlling phenotype expression in BMP-induced osteogenesis and chondrogenesis. Connect Tissue Res. 2002; 43(2-3):529-534.

17. Ayers RA, Simske SJ, Nunes CR, et al. Long-term bone ingrowth and residual microhardness of porous block hydroxyapatite implants in humans. J Oral Maxillofac Surg. 1998; 56(11):1297-1301; discussion 1302.

18. Takabatake $\mathrm{K}$, Yamachika E, Tsuijigiwa $\mathrm{H}$, et al. Effect of geometry and microstructure of honeycomb TCP scaffolds on bone regeneration. J Biomed Mater Res A. 2013; 102(9):2952-2960.

19. Langer R, Vacanti JP. Tissue engineering. Science. 1993; 260(5110):920-926.

20. Tsuruga $\mathrm{E}$, Takita $\mathrm{H}$, Itoh $\mathrm{H}$, et al. Pore size of porous hydroxyapatite as the cell-substratum controls BMP-induced osteogenesis. J Biochem. 1997; 121(2):317-324.

21. Kuboki Y, Takita H, Mizuno M, et al. Geometry of Artificial Extracellular Matrices:a New Paradigm from Dental Tissue Engineering. Dentistry in Japan. 2001; 37:42-50.

22. Jin OM, Takita $\mathrm{H}$, Kohgo $\mathrm{T}$, et al. Effects of geometry of hydroxyapatite as a cell substratum in BMP-induced ectopic bone formation. J Biomed Mater Res. 2000; 51(3):491-499.

23. Kusumbe AP, Ramasamy SK, Adams RH. Coupling of angiogenesis and osteogenesis by a specific vessel subtype in bone. Nature. 2014; 507(7492):323-328.

24. Zigdon-Giladi H, Michaeli-Geller G, Bick T, et al. Human blood-derived endothelial progenitor cells augment vasculogenesis and osteogenesis. J Clin Periodontol. 2015; 42(1):89-95.

25. $\mathrm{Hu}$ J, Srivastava $\mathrm{K}$, Wieland $\mathrm{M}$, et al. Endothelial cell-derived angiopoietin-2 controls liver regeneration as a spatiotemporal rheostat. Science. 2014; 343(6169):416-419.

26. Ding BS, Nolan DJ, Butler JM, et al. Inductive angiocrine signals from sinusoidal endothelium are required for liver regeneration. Nature. 2010; 468(7321):310-315.

27. Murphy MK, Huey DJ, Hu JC, et al. TGF-beta1, GDF-5, and BMP-2 stimulation induces chondrogenesis in expanded human articular chondrocytes and marrow-derived stromal cells. Stem cells. 2015; 33(3):762-773.

28. Sekiya I, Larson BL, Vuoristo JT, et al. Comparison of effect of BMP-2, -4 , and -6 on in vitro cartilage formation of human adult stem cells from bone marrow stroma. Cell Tissue Res. 2005; 320(2):269-276.

29. Hojo H, Ohba S, Taniguchi $K$, et al. Hedgehog-Gli activators direct osteo-chondrogenic function of bone morphogenetic protein toward osteogenesis in the perichondrium. J Biol Chem. 2013; 288(14):9924-9932.

30. Ducheyne P, Radin S, King L. The effect of calcium phosphate ceramic composition and structure on in vitro behavior. I. Dissolution. J Biomed Mater Res. 1993; 27(1):25-34.

31. Scotti C, Tonnarelli B, Papadimitropoulos A, et al. Recapitulation of endochondral bone formation using human adult mesenchymal stem cells as a paradigm for developmental engineering. Proc Natl Acad Sci U S A. 2010; 107(16):7251-7256

32. Fazan F, Marquis PM. Dissolution behavior of plasma-sprayed hydroxyapatite coatings. J Mater Sci Mater Med. 2000; 11(12):787-792.

33. Mate-Sanchez de Val JE, Mazon P, Guirado JL, et al. Comparison of three hydroxyapatite/beta-tricalcium phosphate/collagen ceramic scaffolds: An in vivo study. J Biomed Mater Res A. 2014; 102(4):1037-46.

34. Takeshi U, Naoyuki M, Shunsuke N, et al. Histochemical and Radiological Study of Bone Regeneration by the Combinatorial Use of Tetrapod-Shaped Artificial Bone and Collagen. J Hard Tissue Biology. 2015; 24(2): 199 - 210. 